\title{
Frequency of Cerebrospinal Fluid Otorrhea \& Rhinorrhea among Patients with Traumatic Skull Fracture
}

\author{
Ali Shahjehan', Ali Haider ${ }^{1}$, Hashmat Ullah¹, Khalid Khanzada', Muhammad Akram Khan², \\ Riaz ur Rehman ${ }^{1}$
}

\begin{abstract}
Background: Traumatic Brain Injury (TBI) is a global health issue. TBI is common and has significant societal impact. This type of trauma results in overlying fracture of the skull in $85 \%-95 \%$ of cases.

Objective: To find the frequency of cerebrospinal fluid otorrhea \& rhinorrhea among patients presenting with traumatic skull fracture.

Materials and Methods: This observational descriptive cross section was carried out at neurosurgery department, Lady Reading Hospital Peshawar. Time period of this study was from Jul 12, 2016 to Jan 11, 2017. All patients with age group 18 to 60 years of both gender who presented within 24 hours after the initial head trauma and who were diagnosed with skull bone fracture on $x$ ray were included in the study. On the other hand, patients who developed post-operative cerebrospinal fluid leaks and presented with spontaneous cerebrospinal fluid rhinorrhea or otorrhea or those who developed inflammatory type of Cerebrospinal fluid discharge were excluded from the study.

Results: Total number of patients were 111 with traumatic skull fracture. Among the total patients $72 \%$ were male while $28 \%$ were female. The ratio of male to female was $2.5: 1$. The mean age was 36.13 years $\pm 13.83 S D$ with range of $18-60$ years. $26-40$ years group was common for the traumatic skull fracture in present study. The number of patients with age equal or less than 25 years was $34(30.6 \%)$. Thirty seven (33.3\%) patients were of $26-40$ years, $19(17.9 \%)$ of $40-55$ years and $21(18.9 \%)$ were more than 55 years of age. The Rhinorrhea among traumatic skull fracture was observed in 22(19.8\%) while $89(80.2 \%)$ patients showed no Rhinorrhea. Otorrhea was found in 30(27\%) patients and $1(73 \%)$ patients showed no Otorrhea.

Conclusions: From this study, we concluded that Cerebrospinal fluid otorrhea \& rhinorrhea was the most common complication among patients with traumatic skull fractures.

Key words: Cerebrospinal Fluid, Rhinorrhea Skull Fracture, Otorrhea
\end{abstract}

This article may be cited as: Shahjehan A, Haider A, Ullah H, Khanzada K, Khan MA, Rehman RU. Frequency of Cerebrospinal Fluid Otorrhea \& Rhinorrhea among Patients with Traumatic Skull Fracture.. J Saidu Med Coll Swat 2021;11(4):206-10.DOl:https://doi.org/10.52206/jsmc.2021.11.4.680

\section{INTRODUCTION}

Globally Traumatic brain injury (TBI) is a major health problem, especially in developed countries which is preeminent cause of disability and death in adults and young. While in developing world its incidence is steadily increasing ${ }^{1}$. This traumatic brain injury is classified on the basis of clinical severity, injury mechanis $m$, radiological appearance, distribution (diffuse or focal) or pathology ${ }^{2}$.

$\mathrm{TBI}$ is a common disease and have significant impact on society. According to Center for Disease Control (CDC), at least 2.4 million people were effected with TBI in 2009 among which one third injury were related death containing diagnosis $\mathrm{TBI}^{3,4}$. While about 5.3 million people of US are surviving with $\mathrm{TBI}$ related abnormalities, containing long term and psychological impairments ${ }^{4,5}$. The brain injury caused by

1. Department of Neurosurgery, Lady Reading Hospital, Peshawar Pakistan.

2. Department of Surgery, District Headquarter Hospital Daggar Buner Pakistan.

Correspondence: Dr. Riaz ur Rehman

Neurosurgeon, Lady Reading Hospital Peshawar Pakistan Email: ilyas_khan526@yahoo.com

Received: September 14 ${ }^{\text {th }}, 2020$ Accepted: February 01, 2021 penetration of foreign particles into bony skull and passes into or through the brain substance, which cause the disruption in neurons physiology, fibre tracts, and glial cells. This disruption maybe compounded by haemorrhage and ischaemia ${ }^{6}$ and the most common cause is often blow to the head such as by hammer or baseball bat. This type of injury result in overlying fracture of the skull in $85 \%-95 \%$ of cases. Other types of trauma include road traffic accidents, falls, and assaults, hit by a fast moving object ${ }^{7}$.

Acute or early CSF leaks present within 24 to 48 hours of trauma while the chronic or late leaks present in a delayed fashion such as weeks, months or years later. Most of the cases of traumatic CSF leaks resolve spontaneously with simple conservative measures such as reclined bed position. Current guideline advises conservative treatment of posttraumatic CSF leaks for 10 to 14 days $^{9}$. If the leak does not respond to conservative measures in two weeks, then it is imperative to intervene invasively ${ }^{10-12}$.

The common investigations performed to detect skull fracture and hence the suspected dural tear area is the high resolution CT scan with coronal and sagital cuts. MRI with T2 weighted images in 
prone position is also highly favored. Radionuclide cisternography, metrizamide contrast test and intrathecal fluorescein dye test are other tests to confirm CSF leaks. Beta transferrin of the leaking fluid and its glucose levels are also helpful in guiding to differentiate between CSF rhinorrhoea from other causes ${ }^{12-14}$. Complications of posttraumatic CSF leaks include acute fulminant meningitis with a considerably higher mortality rate, repeated pneumocephalus with the possibility of tension pneumocephalus ${ }^{14-17}$.

The incidence of cerebrospinal fluids leakage follow by craniocerebral injury is 0.5 to $3 \%$, with increasing rate to $25 \%$ for midfacial injuries ${ }^{13}$. In another study, the common complications after TBI were wound infection (13.6\%), neurodeficit (52\%), epilepsy (9 cases) cerebrospinal fluid (CSF) leak $(9.1 \%)$, and post traumatic hydrocephalous (5 patients) $)^{18}$.

International study conducted from 2005-2009, a total of 382,267 patients were analyzed with head injury (Sonig et al. 2012). Among such cases total of $0.2 \%$ (708 patients) was meningitis. While the closed skull fractures $(5.4 \%)$, open skull injury $(0.3 \%)$, cranial vault injury $(1.3 \%)$, and maxillofacid injury (23.2\%) patients were reported. Meningitis was observed in $0.17 \%, 0.18 \%, 0.05 \%$, and $0.10 \%$ in admitted patients respectively. While the cerebrospinal fluid rhinorrhea was found in $453(0.1 \%)$ in head injury patients, and cerebrospinal fluid otorrhea was $582(0.2 \%)$. Among the CSF rhinorrhea patients $7.7 \%$ meningitis was developed, while in rhinorrhea only in $2.6 \%$ meningitis was developed ${ }^{19}$. In another research study, CSF leakage was found in $15 \%$, in such CSF leaking patients otorrhea was found in $62 \%$ and rhinorrhea in $37 \%{ }^{20}$.

CSF leak is a life threatening illness and if not diagnosed and treated in time can lead to poor morbidity and mortality of patients who suffer TBI with skull fracture. This study is designed by keeping in mind the current security situation in our country with ever high rates of blasts and other terrorist activities. In our study if the frequency of CSF leak in term rhinorrhea and otorrhea is found in significant number of patients, then the results of this study will be disseminated to other health professionals and suggestions will be given regarding quick and early screening of all patients who suffer head trauma with skull fracture for CSF leaks.

\section{MATERIALS AND METHOD}

This study was conducted at department of Neurosurgery, Lady Reading Hospital, Peshawar. This was descriptive (cross sectional) study. Sample size was 111 using $37 \%$ prevalence of rhinorrhea in traumatic cerebrospinal fluid leaks ${ }^{20}$, $95 \%$ confidence level and $8 \%$ margin according to WHO formula for sample size calculator. Duration of study was from Jul 12, 2016 to Jan 11, 2017.Sampling technique was Non-probability consecutive. Inclusion criteria was patients with age 18-60 years, Both genders and Patients presenting within 24 hours after the initial head trauma and diagnosed with skull bone fracture on $x$ ray. All those patients who develop postoperative cerebrospinal fluid leaks and Patients who presents with spontaneous cerebrospinal fluid rhinorrhoea or otorrhoea. or those who develop inflammatory type of Cerebrospinal fluid discharge were excluded from the study. The present research work was approved from hospital management and ethical committee. All patients meeting inclusion criteria i.e. all patients with TBI with skull fracture were included in the study through emergency and OPD. The aim of the study was to explain the guardian/relative and informed consent were obtained.

All the patients were worked up with detailed history and clinical examination and diagnosis confirmed using CT and MRI of the brain followed by routine investigations. All patients were carefully examined until 24 hours after admission by a Neurosurgeon for the presence of the CSF leak. The mentioned informations including name, gender, age, and address were registered in a predesigned proforma. For data analysis SPSS 20.0 was used.For the quantitative variables like age, mean \pm standard deviation were calculated. While for the categorical variable such as CSF leakage, rhinorrhea, otorhea, and gender percentage/frequency was calculated. Cerebrospinal fluid leak, otorhhea and rhinorrhea were stratified among gender and age to see effect modifiers. Post stratification was done through chi square test. Keep $P$ value $=0.05$ was considered significant. All the analyzed data were presented in graphs and charts.

Traumatic Skull Fracture ${ }^{3}$ : It was diagnosed among patients with history of trauma to the skull with break in the continuity of skull bone as detected on $\mathrm{X}$ ray skull. 
Cerebrospinal fluid leak ${ }^{2}$ : It was clear water cerebrospinal fluid discharge from the trauma diagnosed on clinical examination 24 hours after presentation with skull fracture.

Rhinorrhea ${ }^{4}$ : Nasal discharge of Cerebrospinal fluid followed by trauma and on clinical examination it was described as a clear fluid discharge through the nose.

Otorrhoea ${ }^{4}$ : Cerebrospinal fluid discharge from the ear and on clinical examination it was described as a clear fluid discharge through the nose.

\section{RESULTS}

In this study total number of patients were 111 with traumatic skull fracture. Among the total patients $72 \%$ were male while $28 \%$ were female. The ratio of male to female was $2.5: 1$ shown in Fig 1. The mean age was 36.13 years $\pm 13.83 S D$ with range of $18-60$ years. The age of patients was categorized into four groups, among which the 2640 years group was common for the traumatic skull fracture in present study. The number of patients with age equal or less than 25 years was $34(30.6 \%)$. Thirty seven (33.3\%) patients were of 26-40 years, 19 (17.9\%), of 40-55 years and $21(18.9 \%)$ were more than 55 years of age as shown in Table 1.

The Rhinorrhea among traumatic skull fracture was observed in 22(19.8\%) while in $89(80.2 \%)$ patients showed no Rhinorrhea. Otorrhea was found in $30(27 \%)$ of patients and $81(73 \%)$ patients showed no Otorrhea.Age wise classification of Rhinorrhea and Otorrhea were found in higher frequency in younger than old age patients. The patients with age equal or less than to 25 years of age had Rhinorrhea of $26.5 \%$ while Otorrhea had the same proportion, age group 26-40 years contained $13.5 \%$ Rhinorrhea and $24.3 \%$ showed Otorrhea, $41-55$ years age groups gave $15.8 \%$ Rhinorrhea and $31.6 \%$ showed Otorrhea, patients with above 55 years had $23.8 \%$ Rhinorrhea and $28.8 \%$ showed Otorrhea in patients with traumatic skull fracture as shown in Table 2.

Gender wise distribution of Rhinorrhea and Otorrhea shows that gender has no role over both although preponderance in male as that of female. There were $21.2 \%$ Rhinorrhea in male and $16.1 \%$ shows in female patients. Similarly on other hand $28.8 \%$ Otorrhea in male and $22.6 \%$ shows in female patients. Table 3 .

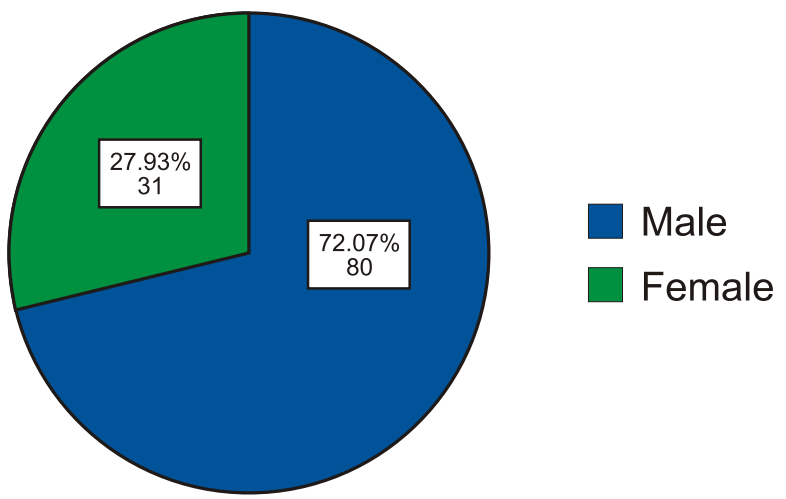

Fig 1. Gender wise distribution of patients

Table 1. Age Wise Distribution of the Patients

\begin{tabular}{|l|c|c|c|}
\hline \multicolumn{1}{|c|}{ Age } & Frequency & Percent & Mean+SD \\
\hline \hline$<=25.00$ & 34 & 30.6 & \\
$26.00-40.00$ & 37 & 33.3 & 36.13 years +13.83 \\
$41.00-55.00$ & 19 & 17.1 & \\
$56.00+$ & 21 & 18.9 & \\
Total & 111 & 100.0 & \\
\hline
\end{tabular}

Table 2. Age Wise Distribution of Otorrhea \& Rhinorrhea Among Patients Presenting with Traumatic Skull Fracture

\begin{tabular}{|l|c|c|c|c|}
\hline \multirow{2}{*}{ Age (in years) } & \multicolumn{2}{|c|}{ Rhinorrhea } & \multicolumn{2}{c|}{ Otorrhea } \\
\cline { 2 - 5 } & Yes & No & Yes & No \\
\hline$<=25$ & $9(26.5 \%)$ & $25(73.5 \%)$ & $9(26.5 \%)$ & $25(73.5 \%)$ \\
$26-40$ & $5(13.5 \%)$ & $32(86.5 \%)$ & $9(24.3 \%)$ & $28(75.7 \%)$ \\
$41-55$ & $3(15.8 \%)$ & $16(84.2 \%)$ & $6(31.6 \%)$ & $13(68.4 \%)$ \\
$56+$ & $5(23.8 \%)$ & $16(76.2 \%)$ & $6(28.6 \%)$ & $15(71.4 \%)$ \\
\hline P-value & $\mathbf{0 . 5 1 7}$ & $\mathbf{0 . 9 4 7}$ \\
\hline
\end{tabular}




\section{Table 3. Gender Wise Distribution of Otorrhea \& Rhinorrhea Among Patients Presenting with Traumatic Skull Fracture}

\begin{tabular}{|c|c|c|c|c|}
\hline & & \multicolumn{2}{|c|}{ Gender } & \multirow{2}{*}{ P-value } \\
\hline & & Male & Female & \\
\hline Rhinorrhea & Yes & 17 & 5 & 0.544 \\
\hline Otorrhea & $\begin{array}{l}\text { Yes } \\
\text { No }\end{array}$ & $\begin{array}{c}21.2 \% \\
63 \\
78.8 \% \\
23 \\
28.8 \% \\
57 \\
71.2 \%\end{array}$ & $\begin{array}{c}16.1 \% \\
26 \\
83.9 \% \\
7 \\
22.6 \% \\
24 \\
77.4 \%\end{array}$ & 0.511 \\
\hline
\end{tabular}

\section{DISCUSSION}

The first study of CSF rhinorrhea after the fracture of traumatic skull and the correlation between them was described by Bidloo in $1745^{7}$. It wasn't studied properly before the advancement in radiography that there is correlation between cranial and nasal cavities diagnosed in patient. The first study about the communication between CSF rhinorrhea and the fracture of traumatic skull was published in 1913 by Luckett $^{15}$. Cairns ${ }^{20}$ classify the CSF rhinorrhea in 1937. He classify it into four classes, i-e; acute traumatic, spontaneous, delayed traumatic, and postoperative. Later, Vrabec and Hallberg ${ }^{21}$ refined this categorization, Ommaya observed $2 \%$ incidence of CSF leakage in all head fractures. This incidence found in $5 \%$ patients when only patients with skull base fractures were considered. Additionally he found that $90 \%$ of CSF leakage was caused by tramatic events. In another study, CSF rhinorrhea can caused from temporal bone injuries, in which $72 \%$ of patients with temporal bone fracture had CSF rhinorrea ${ }^{9,6}$.

In another study approximately 900 patients were operated for head injury, in which 40 patients was operated for frontal DSF. There were 39 men and one woman in this operated patients. The follow up duration was 17.7 months. The causative agent was car and motorcycle incident. The mean period to operate the patients was 3.3 days. Hypotension (4 patients), rhinorrhea (6 patients), otorrhea (4 patients), and hypoxia (2 patients) was observed. Among these patients only 5 patients were died during hospitalization ${ }^{5}$.

CSF leakage was commonly found in $80-90 \%$ cases. Other etiologies contain spontaneous leakage $(3-4 \%)$, postoperative defects $(10 \%)$, inflammation and tumor ${ }^{11}$.

The leakage of CSF will be found in $15-30 \%$ of patients if the head (skull) fracture is happen. This CSF leakage either be immediately or delayed. About $95 \%$ of delayed leakage will be manifest in first 90 days of fracture. Rhinorrheal CSF carries more risk of causing meningitis if it persist for a we $^{9}$. For the prevention of infective complications patients need surgury ${ }^{18}$.

\section{CONCLUSION}

The current study indicated that otorrhea \& rhinorrhea are a common feature in patients with traumatic skull fracture. Continual leakage of CSF for more than $24 \mathrm{hrs}$ are at high risk for causing meningitis, which require surgery. Treatment should be directed according to the severity of neurological slump in emergency time and association of intracranial injury presence or absent. The period of operating and CSF drainage must be decided carefully.

\section{CONFLICT OF INTEREST}

The author declares no conflict of interest in this article. 


\section{REFERENCES}

1. Barkhoudarian G, Houda DA. The molecular pathophysiology of concussive brain injury. Clin Sports Med. 2017;30:33-48.

2. Risdall JE, Menon DK. Traumatic brain injury. Philosophical Transactions of the Royal Society B: Biological Sciences, 2018;366(1562):241-50.

3. Coronado VG, McGuire LC, Sarmiento K, Bell J, Lionbarger MR, Jones CD, et al. Trends in traumatic brain injury in the US and the public health response: 19952009. J safety res 2018;43(4):299307.

4. Brooks JC, Strauss DJ, Shavelle RM, Paculdo DR, Hammond FM, Harrison-Felix CL. Long-term disability and survival in traumatic brain injury: Results from the National Institute on Disability and Rehabilitation Research Model Systems. Arch physical med rehabilit. 2018;94(11):2203-9.

5. Kurland D, Hong C, Aarabi B, Gerzanich V, Simard JM. Hemorrhagic progression of a contusion after traumatic brain injury: a review. J neurotrauma. 2019;29(1):19-31.

6. Chen S, Xu C, Yuan L, Tian H, Cao H, Guo Y. Fatal deterioration of delayed acute subdural hematoma after mild traumatic brain injury: two cases with brief review. Chinese J Traumatol. 2020;17(2):115-7.

7. Kumar BR, Sahu R, Srivastava AK, Nair AP, Mehrotra A. Surgically repaired posttraumatic CSF rhinorrhea: An institutional experience and review of literature. Indian J Neurosurg. 2017; 1:23-7.

8. Kim SW, Park HW, Jeon SY. Versatility of the pedicled nasoseptal flap in the complicated basal skull fractures. Auris Nasus Larynx. 2018 Jun;40(3):334-7.

9. Tahir MZ, Khan MB, Bashir MU, Akhtar S, Bari E. Cerebrospinal fluid rhinorrhea: An institutional perspective from Pakistan. Surg Neurol Int. 2019;2:174-79.

10. Aurangzeb A, Ahmed E, Khan SA. Outcome of transcranial repair of traumatic CSF rhinorrhoea. J Ayub Med Coll Abbottabad. 2017;24(2):47-9.

11. Bhatti SN, Khan SA, Shah R. Transnasal endoscopic repair of cerebrospinal fluid rhinorrhoea. J Ayub Med Coll Abbottabad. 2018;23(2):15-7

12. Chaudhary N, Awan LM, Niaz A. Success determination of lumber drainage in cranial traumatic CSF fistula. J Spine Neurosurg. 2017;2:511.

13. Khan Z, Ali M, Sharafat S, Usman M, Aman R, Khan $\mathrm{KM}$, et al. Bomb blast head injuries: a two years' experience of 154 patients Rawal Med J. 2018;37(4):417-20.

14. Janfaza P. Surgical Anatomy of the Head and Neck. Philadelphia, Pa: Lippincott Williams \& Wilkins;2019.p.481-505.

15. Lyons BM. Surgical anatomy of the skull base. Donald PJ. Surgery of the Skull Base. LippincottRaven;2019.p.15-30.

16. Nuss DW, O'Malley BW. Surgery of the anterior and middle Cranial Base. Cummings CW. Cumming's Otolaryngology Head and Neck Surgery. 4th ed. St Louis, Mo: Elsevier Mosby;2019.p.3760-75.

17. Tiedemann K. Gross sectional anatomy. Janecka IP. Skull Base Surgery: Anatomy, Biology, and Technology. Philadelphia, Pa: LippincottRaven;2018.p.75-149.
18. Karasu A, Cansever T, Batay F, Sabanci PA, AlMefty $O$. The microsurgical anatomy of the hypoglossal canal. Surg Radiol Anat. 2009;31(5):363-7

19. Flint PW. CHAPTER 173 Surgical Anatomy of the Lateral Skull Base. Cummings Otolaryngology Head and Neck Surgery. 5th. Philadelphia, PA: Elsevier; 2019. chap 173.

20. San Millan Ruiz D. Anomalous Intracranial Drainage of the Nasal Mucosa: A Vein of the Foramen Caecum?. Am J Neuroradiol. 2016 Jan;27:129-31.

21. Atta HM. Edwin Smith Surgical Papyrus: the oldest known surgical treatise. Am Surg. 2019 Dec 65(12):1190-2.

DATA SHARING STATEMENT: The data that support the findings of this study are available on request from the corresponding author. The data are not publicly available due to privacy or ethical restrictions.

CONFLICT OF INTEREST: Authors declared no conflict of interest.

GRANTED SUPPORT AND FINANCIAL DISCLOSURE: Nil

\section{AUTHOR'S CONTRIBUTION}

Following authors have made substantial contributions to the manuscript as under

Shahjehan A:

Concept and design of study, Collection of data, statistical analysis

Haider A, Ullah H: Writing of manuscript, critical review of manuscript

Khanzada K, Khan MA: Analysis and interpretation of $\begin{array}{ll} & \text { data, statistical analysis } \\ \text { Rehman UR: } & \text { Data collection, bibliography }\end{array}$

Authors agree to be accountable for all aspects of the work in ensuring that questions related to the accuracy or integrity of any part of the work are appropriately investigated and resolved. 